\section{REMOCIÓN DE UN INSTRUMENTO FRACTURADO DURANTE LA TERAPIA ENDODÓNTICA: REPORTE DE UN CASO}

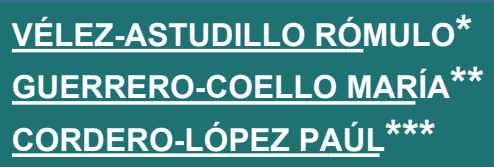

Recibido: 11/08/2015

Aceptado: 08/11/2015

\title{
RESUMEN
}

Durante los procedimientos de preparación del conducto radicular, el potencial de fractura de instrumentos está siempre presente. Cuando un instrumento se fractura, produce ansiedad al tratante, así como, una obstrucción metálica del conducto lo que dificulta aún más la limpieza y conformación. Se debe intentar siempre remover el instrumento fracturado, y si la recuperación no es posible; se debe evaluar la posibilidad de sobrepasarlo.

El propósito de este artículo es reportar un caso clínico de remoción de un instrumento endodóntico fracturado en el interior del conducto radicular, mediante la creación de un espacio alrededor del instrumento y con la ayuda de dos limas Hedström que traccionan de él, removiéndolo por completo.

Palabras clave: Endodoncia, instrumento, fractura, Fresa separada, resultado.

\section{ABSTRACT}

During root canal preparation procedures, the potential for instrument breakage is always present. When instrument breakage occurs, it leads to anxiety of the clinician as well as a metallic obstruction of the canal which hinders further cleaning and shaping. Separated instruments must always be attempted for retrieval and if retrieval is not possible bypass should be tried. The purpose of this work is to report a clinical case of removal of a fractured endodontic instrument in the root canal by creating a space around the fractured instrument and then engaging it with the help of two Hedström files by twisting around the fractured instrument and then pulling it out.

Key words: Endodontics, Instrument, fracture, Separated Bur, outcome

\section{INTRODUCCIÓN}

La fractura de un instrumento dentro del conducto radicular durante el tratamiento endodóntico no es un incidente raro; en varios estudios retrospectivos revisados por Hülsmann y Schinkel ${ }^{1}$, la evaluación de radiografías indicó que la frecuencia de fragmentos de instrumentos remanentes en el conducto estaba entre el 2 y $6 \%$, aunque solo provoca un fracaso del $1 \%$ de los tratamientos ${ }^{2}$. Durante los procedimientos endodónticos, la potencial fractura de un instrumento siempre está presente. Muchos odontólogos asocian "instrumentos rotos" con limas, pero este término también puede ser aplicado a puntas de plata, fresas Gate Gliddens, léntulos y condensadores ${ }^{3}$.

Aunque la fractura del instrumental puede ser consecuencia de un defecto de fabricación, la causa más habitual es el empleo inadecuado de los mismos por parte del odontólogo, que fuerza el instrumento o prolonga su uso más allá de su vida útil ${ }^{3}$. Se debe tener en cuenta que las propiedades físicas de un instrumento, se van deteriorando, tanto con el uso y con los continuos y bruscos cambios de temperatura al esterilizarlos ${ }^{4}$. Lasala refiere que en el momento de rectificar la apertura de cámara y el acceso a los conductos, se puede producir la fractura de las fresas; esto ocurre posiblemente debido a la profundidad del tejido, la forma de la fresa y a la activación de la misma cuando ya está colocada en posición, en lugar de llevarla activada al punto de trabajo 4 .

En la literatura científica se han descrito varios protocolos de tratamiento para estos accidentes del procedimiento, lográndose diferentes niveles de éxito, incluyendo los siguientes: el instrumento fracturado deberá extraerse, dejarse en el conducto y ser sobrepasado e incorporado en la obturación final del conducto radicular; o se usarán técnicas quirúrgicas para retirar el objeto o la totalidad de la porción de la raíz que aloje al mismo ${ }^{5}$.

Sin embargo, aun cuando la sustracción del instrumento se lleve a cabo con éxito, ciertas complicaciones surgidas durante el procedimiento de remoción, pueden resultar en considerable pérdida de estructura dental, así como perforaciones radiculares ${ }^{6,7}$. Por lo tanto, resulta muy importante evaluar la complejidad inherente al retirar los fragmentos de instrumentos, así como los posibles efectos desfavorables de estas complicaciones del procedimiento.

* Odontólogo egresado, Universidad Católica de Cuenca.Ecuador

** Docente Especialista en Endodoncia, Universidad Católica de Cuenca.Ecuador

*** Docente Especialista en Rehabilitación Oral, Universidad Católica de Cuenca.Ecuador 
El propósito del presente artículo es informar sobre un caso de fractura y remoción de una fresa Endo Z, durante el acceso cameral de un incisivo lateral superior izquierdo.

\section{REPORTE DEL CASO}

Paciente masculino de 15 años de edad, acude a la Clínica de Odontología de la Universidad Católica de Cuenca por motivo estético de sus dientes anterosuperiores. Los antecedentes médicos del paciente no revelaron información pertinente al caso. Al examen clínico intraoral se diagnosticó caries en diferentes piezas dentarias, entre ellas: caries proximal en el incisivo lateral superior izquierdo (2.2). Se procedió a realizar las pruebas de vitalidad pulpar en la pieza 2.2 que resultaron negativas. A la percusión vertical y palpación hubo respuesta positiva, así como se observó una fístula activa en relación al ápice de la pieza dentaria.

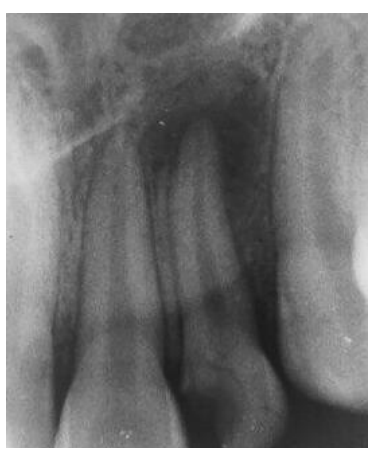

Figura 1. Radiografía periapical ensanchamiento del ligamento periodontal y lesión radiolúcida circunscrita alrededor del ápice
Al examen radiográfico se consideró la cámara y conducto radicular como amplios, se observó ensanchamiento del ligamento periodontal y lesión radiolúcida circunscrita alrededor del ápice (Fig. 1). Se llegó al diagnosticó pulpar y periodontal compatible con necrosis pulpar y absceso periapical crónico respec-tivamente. Asimismo, se le sugirió al paciente un tratamiento endodóntico.

Después de administrar la anestesia local (lidocaína 2\% con epinefrina 1:80.000, New Stetic, Colombia), se inició con la terapia endodóntica y la perforación inicial se realizó con una fresa de diamante redonda de cuello largo mediana (Fava).

Posteriormente para dar la forma de conveniencia al acceso cameral, se utilizó una fresa de carburo endo Z (SS White), la cual se fracturó y quedó atrapada en el interior del conducto.

Mediante una radiografía periapical, se constató la longitud del fragmento y la localización del mismo en el interior del conducto (Fig.2). Se observó que el instrumento separado tuvo una longitud aproximada de $8 \mathrm{~mm}$ y se localizó a nivel de los tercios cervical y medio del conducto radicular.

Debido al diagnóstico de la pieza dentaria y al mal pronóstico que representaría el hecho de no retirar el instrumento fracturado, se decidió removerlo de la siguiente manera:

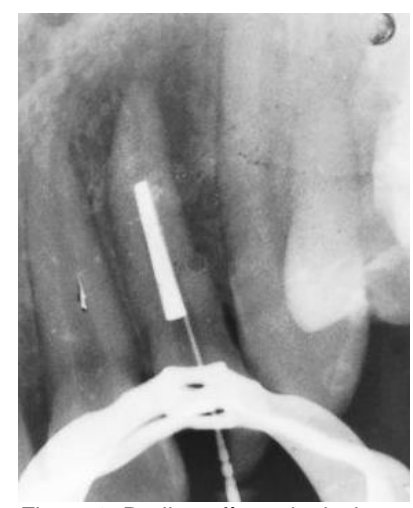

Figura 2. Radiografía periapical longitud del fragmento y localización del mismo

Con la ayuda de la fresa Gates Glidden número 1 (Dentsply Maillefer), se creó un espacio alrededor del instrumento para poder observarlo de mejor manera y posteriormente se lo sobrepasó con limas $\mathrm{K}$ número 15 y 20 (Dentsply Maillefer). Se colocaron dos limas Hedstrom número 15 y 20 (Dentsply Maillefer) alrededor de la fresa separada en distintos lados y se realizó un movimiento de tracción hacia coronal, que permitió que el instrumento sea removido del interior del conducto radicular (Fig. 3).

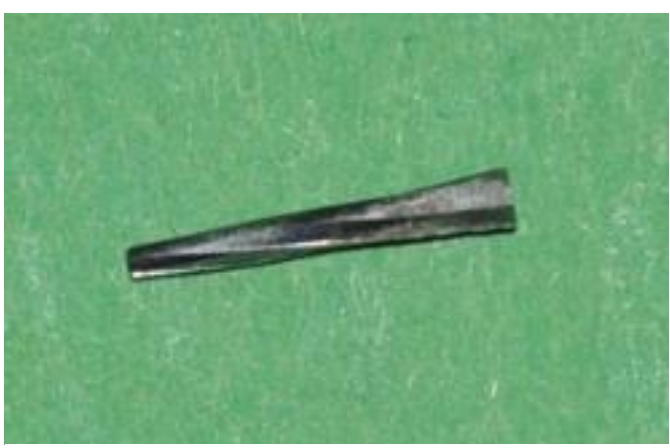

Figura 3. Instrumento removido del interior del conducto radicular
Se realizó una radiografía periapical para confirmar que el instrumento haya sido removido en su totalidad. Con la ayuda de un localizador de ápice (Propex Pixi, Dentsply Maillefer) se registró la longitud de trabajo y se realizó la preparación biomecánica pertinente. Posteriormente, el conducto recibió medicación por 30 días (polvo de hidróxido de calcio puro y solución salina); con un recambio a los 15 días. Se finalizó la terapia endodóntica con la obturación del conducto radicular. 
Se tomó una radiografía postoperatoria para evaluar la calidad de la obturación del conducto (Fig. 4.). El paciente no experimentó dolor u otras complicaciones postoperatorias, por lo que en una cita después de dos meses se llevó a cabo una restauración coronaria apropiada para asegurar el sellado adecuado (Fig. 5,). La radiografía de control tomada a los 4 meses mostró mejoría en los tejidos periradiculares, además; el paciente se presentó asintomático. El paciente será objeto de monitoreo a los 6, 9 y 12 meses para evaluar las respuestas periradiculares.

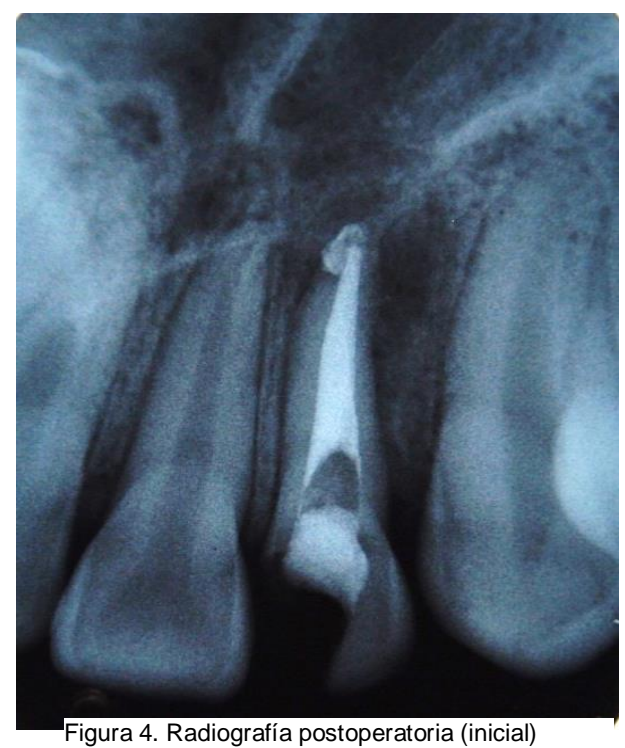

\section{DISCUSION}

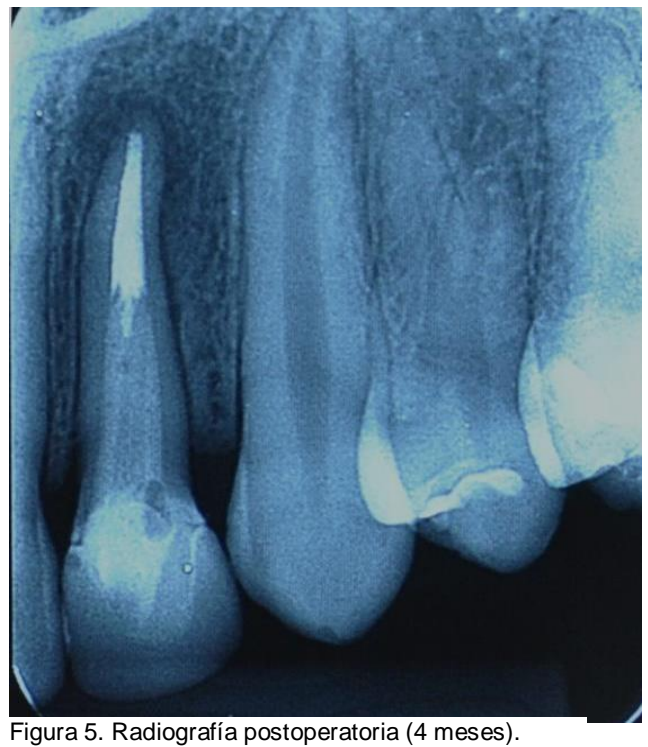

Figura 5. Radiografía postoperatoria (4 meses).

Se han planteado diversas soluciones para tratar la fractura de instrumentos, dependiendo del momento en que se fracturó, del nivel en el que se encuentra el instrumento dentro del sistema de conductos y del tipo de instrumento fracturado Lasala ${ }^{4}$ y Ruíz et al. ${ }^{8}$. En cuanto al momento en que ocurrió la fractura de un instrumento ya sea este al inicio o al final de la preparación biomecánica, el mismo presenta retos diferentes, ya que varía la cantidad de tejido pulpar contenido en los conductos ${ }^{4}$.

Varias técnicas se han propuesto para la remoción de instrumentos fracturados. Con respecto al empleo de pinzas especiales como la pinza de Steiglitz (Moyco, Union Broach, York, Penn), Lovdahl et al. ${ }^{9}$ refieren que las mismas funcionan en contadas ocasiones, sin embargo no las recomiendan en todos los casos, ya que el instrumento debe ser muy largo para poder tomarlo y las estrías de la pinza no están diseñadas para atrapar el fragmento.

En cuanto al equipo de Masserann (Micromega SA, Bensacon, France), Lovdahl et al. ${ }^{9}$ lo recomiendan principalmente para la remoción de puntas de plata y pernos, aunque puede ser utilizado en determinados casos de instrumentos fracturados.

Hulsmann ${ }^{10,11}$ recomienda sobrepasar o remover el instrumento fracturado utilizando el sistema Canal Finder (Fa. Societe Endo Tecnique, Marseille France) y aseguran que puede lograrse en el $50 \%$ de los casos, donde la remoción manual ha fallado. De manera contradictoria el autor refiere cierto riesgo de producir perforaciones cuando se utiliza el sistema a alta velocidad.

Los aparatos ultrasónicos se han usado ampliamente en la remoción de instrumentos fracturados y cuentan con dispositivos variados que pueden facilitar la remoción de los mismos ${ }^{10,12}$. Suter ${ }^{13}$ recomienda una técnica donde utiliza puntas ultrasónicas para liberar la porción coronaria del instrumento y una aguja desechable y limas Hedström para removerlos del conducto.

Ruiz et al. ${ }^{14}$ y Walvekar et al..$^{15}$ establecen que se puede extraer una lima, si es sobrepasada con otra y traccionada hacia afuera friccionando sobre la misma; se debe tener especial cuidado al momento de sobrepasarla ya que un movimiento brusco puede desplazarla en sentido apical, complicando la situación aún más. Igualmente, se puede extraer el fragmento utilizando dos limas Hedström en lados opuestos del instrumento fracturado, para arrastrar el fragmento hacia coronal, después de haberlo sobrepasado con limas finas tipo K. Este principio coincide con el caso clínico citado, puesto que se utilizó dos limas Hedström en distintos lados del instrumento fracturado y posteriormente se realizó un movimiento de tracción, lo cual logró 
remover el instrumento hacia afuera. Aunque de manera contraria, Lovdahl et al. ${ }^{9}$ refieren que las limas Hedström no pueden enganchar instrumentos de acero y por lo tanto no funcionan en la remoción de instrumentos fracturados, léntulos o fresas Gates Glidden.

Por otro lado, la evidencia indica que, el pronóstico de los tratamientos endodónticos en los casos donde se dejan fragmentos de instrumentos al interior del conducto radicular no se ve afectado 5 .

El pronóstico será menos favorable en aquellos casos que presenten enfermedad periapical al momento del tratamiento. Un hallazgo observado en este reporte de caso clínico fue la presencia de lesión periapical preoperatoria, factor que sirvió para manejar el caso. Después de producirse la fractura de la fresa, basándose en un diagnóstico de necrosis pulpar, así como absceso apical crónico, se decidió retirar el instrumento ya que si no se realizaba este procedimiento; bloquearía la posibilidad de una adecuada limpieza, preparación y obturación del conducto Glickman et ${ }^{16}$.

Hasta la fecha, la mejor evidencia con respecto al pronóstico de instrumentos fracturados retenidos, proviene de series de estudios de casos, los cuales a su vez ofrecen bajo nivel de evidencia. Sólo se han identificado dos investigaciones auténticas de control de casos 17,18. Los resultados de los tratamientos no han experimentado cambios significativos en las últimas décadas ${ }^{19}$.

Por lo tanto, es necesario dar seguimiento apropiado de los pacientes en caso de existir cualquier complicación clínica; esto permite la revisión periódica y evaluación radiográfica para prever y combatir cualquier problema potencial. En casos donde se detecte el deterioro de la salud periapical, deberán contemplarse procedimientos de cirugía apical o extracción ${ }^{20}$. El seguimiento en este caso clínico fue a los 4 meses después de obturar el conducto radicular, en donde el paciente se presentó asintomático, así como se percibió mejoría de la región periapical.

\section{CONCLUSIÓN}

Con el método alternativo, que implica el uso de dos limas Hedström se logró retirar correctamente el fragmento de instrumento fracturado dentro del conducto radicular. Este método puede ser utilizado por cualquier odontólogo general o especialista que no posea un sistema específico para la remoción de instrumentos fracturados. El método es simple, rentable y al mismo tiempo puede resultar exitoso.

Se requieren estudios futuros para evaluar la efectividad de las diferentes técnicas y determinar el mejor procedimiento para remoción de instrumentos retenidos en el interior del conducto.

\section{REFERENCIAS BIBLIOGRÁFICAS}

1. Hulsmann M, Schinkel I. Influence of several factors on the success or failure of removal of fractured instruments from the root canal. Endod Dent Traumatol. 1999 Dec; 15(6):252-8.

2. Rubio D. Sistemas para la extracción de instrumentos separados en el interior de conductos radiculares. Dental Practice Report. Marzo 2008: 42-45.

3. Ruddle C. Retratamiento endodóncico no quirúrgico. En: Vías de la Pulpa. 8va ed. Madrid: Cohen S, Burns R. editores. Editorial Mosby; 2002.

4. Lasala A. Endodoncia.4ta ed. México, Salvat, 1993.

5. Panitvisai $\mathrm{P}$, Parunnit $\mathrm{P}$, Sathorn $\mathrm{C}$, Messer $\mathrm{HH}$. Impact of a retained instrument on treatment outcome: a systematic review and meta-analysis. J Endod. 2010; 36 (5): 775-780.

6. Souter $\mathrm{NJ}$, Messer $\mathrm{HH}$. Complications associated with fractured file removal using an ultrasonic technique. J Endod. 2005; 31 (6): 450452. 
7. Ward JR, Parashos $\mathrm{P}$, Messer HH. Evaluation of an ultrasonic technique to remove fractured rotary nickeltitanium endodontic instruments from root canals: clinical cases. J Endod. 2003; 29 (11): 764-767.

8. Ruiz P, San Martín S. Fractura de lima: posibilidades terapéuticas. Endodoncia 1998; 16(3):172-9.

9. Lovdahl PE, Gutmann JL. Problems in non-surgical root canal retreatment. En: Gutmann JL, Dumsha TC, Lovdahl PE, Hovland EJ, editors. Problem solving in endodontics. Missouri. Mosby, 1997:157-201.

10. Hülsmann M. The removal of silver cones and fractured instruments using the canal finder system. J Endodon 1990; 16(12)596-600.

11. Hülsmann M. Removal of fractured instruments using a combined automated/ultrasonic technique. J Endodon 1994; 20(3)144-6.

12. Lovdahl PE, Wade CK. Problems in tooth isolation and periodontal support for the endodontically compromised tooth. En: Gutmann JL, Dumsha TC, Lovdahl PE, Hovland EJ, editors. Problem solving in endodontics. Missouri. Mosby, 1997:203-27.

13. Stabholz A, Friedman S. Tamse A. Endodontic failures and re-treatment. En: Cohen S, Burns RC, editors. Pathways of the pulp. Missouri. Mosby, 1994:709-28.

14. Ruiz P, Vega JM, Zabalegui B, García J. Fracturas de instrumentos en el interior de los conductos radiculares: Casos clínicos. Endodoncia 1998; 16(3):127-31.

15. Walvekar SV, Al\&endash; Duwain Y, Al-Kandasi AM, Al Quond OM. Unusual foreign objects in the root canal. J Endod. 1995; 21(10):526-7.

16. Glickman GN. Problems in canal cleaning and shaping. En: Gutmann JL, Dumsha TC, Lovdahl PE, Hovland EJ, editors. Problem solving in endodontics. Missouri. Mosby, 1997:91-121.

17. Crump MC, Natkin E. Relationship of broken root canal instruments to endodontic case prognosis: a clinical investigation. J Am Dent Assoc. 1970; 80: 1341-1347.

18. Spili $\mathrm{P}$, Parashos $\mathrm{P}$, Messer $\mathrm{HH}$. The impact of instrument fracture on outcome of endodontic treatment. J Endod. 2005; 31: 845-850.

19. Friedman S. Expected outcomes in the prevention and treatment of apical periodontitis. In: Ørstavik D, Pitt Ford TR, eds. Essential endodontology: prevention and treatment of apical periodontitis. 2nd ed. Oxford: Blackwell Munksgaard Ltd; 2008. pp. 408-469.

20. Madarati AA, Watts DC, Qualtrough AJ. Opinions and attitudes of endodontists and general dental practitioners in the UK towards the intra-canal fracture of endodontic instruments: part 2. Int Endod J. 2008; 41: 1079-1087. 